Les cours comme lieu de rencontre et d'élaboration des langues vernaculaires à la Renaissance (1480-1620), J. Balsamo, A. K. Bleuler (éds.)

\title{
Alessandro Bertolino
}

\section{(2) OpenEdition}

\section{Journals}

Edizione digitale

URL: http://journals.openedition.org/studifrancesi/9927

DOI: $10.4000 /$ studifrancesi.9927

ISSN: 2421-5856

\section{Editore}

Rosenberg \& Sellier

\section{Edizione cartacea}

Data di pubblicazione: 1 août 2017

Paginazione: 350-351

ISSN: 0039-2944

\section{Notizia bibliografica digitale}

Alessandro Bertolino, «Les cours comme lieu de rencontre et d'élaboration des langues vernaculaires à la Renaissance (1480-1620), J. Balsamo, A. K. Bleuler (éds.)», Studi Francesi [Online], 182 (LXI | II) | 2017, online dal 01 août 2017, consultato il 07 janvier 2021. URL: http://journals.openedition.org/ studifrancesi/9927 ; DOI: https://doi.org/10.4000/studifrancesi.9927

Questo documento è stato generato automaticamente il 7 janvier 2021.

\section{cc) (†)}

Studi Francesi è distribuita con Licenza Creative Commons Attribuzione - Non commerciale - Non opere derivate 4.0 Internazionale. 


\title{
Les cours comme lieu de rencontre et
} d'élaboration des langues vernaculaires à la Renaissance (1480-1620), J. Balsamo, A. K. Bleuler (éds.)

\author{
Alessandro Bertolino
}

\section{NOTIZIA}

Les cours comme lieu de rencontre et d'élaboration des langues vernaculaires à la Renaissance (1480-1620) / Höfe als Laboratorien der Volkssprachigkeit zur Zeit der Renaissance (1480-1620), coordination éditoriale / herausgegeben von Jean BALSAMO - Anna Kathrin BLEULER, Genève, Droz, 2016, «Travaux d'Humanisme et Renaissance» 565, 471 pp.

1 Dopo i due volumi dedicati agli ateliers di stampa e alle città come luoghi di promozione e sviluppo del volgare, la serie «De lingua et linguis», che presenta i risultati del progetto franco-tedesco Eurolab sull'emergenza delle lingue vernacolari, si arricchisce di una nuova raccolta di contributi che indagano il ruolo svolto dalle corti in questo importante fenomeno del Rinascimento europeo. In omaggio alla natura binazionale del progetto, i testi introduttivi sono presentati in francese e in tedesco e gli abstract dei singoli contributi sono nelle due lingue. Nell'Introduction / Einführung, Jean BALSAMO e Anna Kathrin BLEULER ricordano come proprio l'ambiente curiale - sede della concentrazione del potere e luogo di incontro tra varie nazionalità - costituisca uno spazio privilegiato sia per la promozione di una lingua nazionale da intendersi come espressione del prestigio politico, sia per un confronto tra diverse tradizioni linguistiche coesistenti e talvolta concorrenti. Inoltre, i due curatori del volume distinguono tra un multilinguismo di corte («Mehrsprachigkeit»), ovvero la presenza simultanea di lingue diverse per esigenze pratiche o a causa della composizione plurinazionale dell'entourage del principe, e un plurilinguismo («Vielsprachigkeit»), 
risultato di una politica attiva del principe a favore dello sviluppo di una cultura di corte che si esprima in più lingue. Questa distinzione terminologica determina anche la struttura del volume, che si presenta articolato in tre sezioni: la prima indaga la pratica multilingue di varie corti europee; la seconda riunisce contributi relativi alla poesia di corte, principale forma di espressione di una cultura plurilingue; la terza infine si concentra sull'attività delle accademie e dei circoli letterari, fisicamente separati dalla corte, ma dipendenti da essa per la loro esistenza. All'Avant Propos / Vorwort (pp. 7-8) dei due responsabili del progetto, Elsa KAMMERER e Jan-Dirk MÜLLER, e all'Introduction già citata (pp. 9-47), seguono i contributi sotto elencati, per la quasi totalità già presentati nel corso del seminario Eurolab tenutosi a Monaco di Baviera il 7-8 settembre 2012, con l'eccezione degli studi di Piotr Salwa, Aude Plagnard e Anna Kathrin Bleuler.

2 Première partie («Une pratique de cour: le multilinguisme / Pragmatische Mehrsprachigkeit an Höfen»): Jan-Dirk MÜLLER, Mehrsprachigkeit am Kaiserhof, pp. 51-69; Regina BAAR-CANTONI, Skizze eines «Laboratoriums der Volkssprachigkeit»: Der Hof Kurfürst Friedrichs II. von der Pfalz als Ort kulturellen Austauschs, pp. 71-89; Roland BÉHAR, Multilinguisme à la cour du vice-roi de Naples don Pedro de Toledo (1532-1553), pp. 91-134; Piotr SALWA, Le multilinguisme de la cour polonaise à l'époque de la Renaissance. Esquisse préliminaire, pp. 135-151; Anders TOFTGAARD, Les langues à la cour de Danemark à la Renaissance et l'italianisme à l'époque de Christian IV (1577-1648), pp. 153-180; Véronique FERRER, D'Aubigné et la satire du gascon à la cour de France, pp. 181-193. Deuxième partie («La poésie, lieu du plurilinguisme de cour / Hofdichtung als Kontaktzone von Vielsprachigkeit»): Johannes Klaus KIPF, Fabian PRECHTL, Volkssprachige Versuche in München und Augsburg zwischen Hof, Schule und Universität (1480-1620), pp. 197-240; Anna Kathrin BLEULER, Deutschsprachige Hofdichtung zur Zeit Friedrichs II. von der Pfalz (1520-1556), pp. 241-269; Aude PLAGNARD, Le portugais et la cour des Habsbourg d'Espagne: usages nobiliaires, circulations écrites et pratiques littéraires, pp. 271-295; Franco TOMASI, Les poètes italiens à la cour de France, pp. 297-315; Daniele SPEZIARI, Le plurilinguisme funèbre autour de Marguerite de France, duchesse de Berry et de Savoie, pp. 317-332. Troisième partie («Académies, sociétés et réseaux lettrés / Sprachakademien und gelehrte Netzwerke»): Klaus CONERMANN, Das Deutsche und die Vielsprachigkeit in der Frühzeit der Fruchtbringenden Gesellschaft. Der Köthener Hof als Laboratorium der Sprach- und Versarbeit, pp. 335-380; Sarah DESSì SCHMID, Jochen HAFNER, Die italienischen und französichen Akademien als Zentren frühneuzeitlicher höfischer Sprachdiskussion, pp. 381-418. English abstracts, pp. 419-445; Index, pp. 447-465.

3 I contributi dedicati espressamente al caso francese sono poco numerosi, ma di notevole interesse. Franco TOMASI ritraccia la presenza della poesia in lingua italiana alla corte di Francesco I: partendo dalla definizione di lingua cortigiana formulata da Castiglione nel suo dialogo, Tomasi individua nell'apertura verso la lingua italiana - in grado di fornire esempi di un "classicisme moderne et complexe» (p. 305) - uno dei tratti distintivi della politica culturale del sovrano francese. Se l'attenzione di Francesco I nei confronti della poesia della Penisola nasce da un interesse personale, come testimoniano le traduzioni e gli adattamenti di mano del sovrano, la trasformazione di questo interesse nel progetto di un «italianisme royal» (p. 303), che informa la cultura di corte, segue delle tappe ben precise, la prima delle quali è la costruzione di un culto di Petrarca, tramite le traduzioni e le imitazioni della sua opera, ma anche attraverso quel grande evento simbolico che fu la "scoperta" della tomba di Laura, e che espresse - a qualche settimana dal matrimonio tra Henri de Valois e 
Caterina de' Medici - la volontà di legare la fama del poeta laureato alla terra di Francia. Rispondono a una logica simile anche i due volumi delle Opere toscane di Luigi Alamanni, fortemente voluti dalla corte, alla quale offrono un esempio di varie forme poetiche (lirica, elegia, poesia bucolica e sacra) tratte dalla tradizione classica ma rivisitate in una lingua volgare moderna (p. 306). Questo italianismo reale non si riduce tuttavia a un'importazione del modello d'oltralpe, ma orienta la produzione dei poeti italiani a corte parallelamente allo sviluppo della letteratura francese: con la composizione di Girone il cortese, l'Alamanni si inserisce infatti nel tentativo di recupero delle antiche storie cavalleresche, iniziato già con le traduzioni degli Amadigi e di Ariosto; pochi anni più tardi, Bartolomeo Del Bene tenterà con i suoi versi italiani di allinearsi all'esperienza poetica della Pléiade. Il contributo di Véronique FERRER mostra invece la valenza politica del gioco linguistico a corte, tramite l'esempio di un romanzo d'Agrippa d'Aubigné, Les avantures du Baron de Fanaeste, che attraverso l'utilizzo di un francese con forti tratti guasconi punta a polemizzare con la corte di Enrico IV e la reggenza di Maria de' Medici, di cui denuncia indirettamente la decadenza morale (p. 190); parallelamente, il personaggio del parvenu guascone, con una lingua mista di dialettalismi e langage de cour, diventa simbolo di tutta una categoria sociale, quella dei protestanti che rinnegano la loro fede per poter entrare a corte. Alla questione religiosa fa riferimento anche SPEZIARI, analizzando le modalità con cui sono costruiti due tombeaux plurilingue dedicati a Margherita di Francia, duchessa di Berry e di Savoia. Emerge chiaramente come nel Tombeau de Marguerite de Valoys Royne de Navarre (1551), la compresenza di versi in latino, greco e francese (nonché, ma in misura minore, in italiano) cerchi di instaurare un parallelo - o una competizione - tra la poesia francese e il modello classico, ad esempio attraverso traduzioni concorrenti degli stessi versi latini; il gruppo di poeti che si mette alla prova in questa raccolta, coordinata da Nicolas Denisot, mira a presentare quindi un modello di poesia umanista, da mettere sotto la protezione della nipote della defunta. All'opposto, l'Ombre et tombeau (1574) composto per la morte della "seconda Margherita" presenta un sostanziale bilinguismo latino-francese (con ridottissimi elementi in italiano o in greco), in cui però è assente la dinamica della traduzione; l'anonimato dei contributori (tra i quali potrebbero nascondersi Odet de La Noue e Jacques Davy du Perron), i riferimenti alla fede riformata e l'utilizzo del francese per lanciare un messaggio militante inducono a vedere questa raccolta come l'omaggio di una «cour secrète» (p. 327) protestante alla sua principessa. Infine, DESSì SCHMID e HAFNER tracciano un'interessante storia dell'Académie française, ricordando gli stretti rapporti tra la Corte e questa istituzione, fondata nel 1635 da Richelieu - ma i cui antecedenti sono nei réseaux lettrés del XVI secolo - nonché il rapporto con le accademie provinciali, anch'esse legate al potere reale. 